\title{
BENCHMARKING LEARNER EDUCATION USING ONLINE
}

\section{BUSINESS SIMULATION}

\author{
Alfred Miller \\ Fujairah Women's College UAE \\ Box 1626 Fujairah, UAE \\ amiller@hct.ac.ae
}

\begin{abstract}
For programmatic accreditation by the Accreditation Council of Business Schools and Programs (ACBSP), business programs are required to meet STANDARD \#4, Measurement and Analysis of Student Learning and Performance. Business units must demonstrate that outcome assessment systems are in place using documented evidence that shows how the results are being used to further develop or improve the academic business program. The Higher Colleges of Technology, a 17 campus federal university in the United Arab Emirates, differentiates its applied degree programs through a 'learning by doing ethos,' which permeates the entire curricula. This paper documents benchmarking of education for managing innovation. Using business simulation for Bachelors of Business, Year 3 learners, in a business strategy class; learners explored through a simulated environment the following functional areas; research and development, production, and marketing of a technology product. Student teams were required to use finite resources and compete against other student teams in the same universe. The study employed an instrument developed in a 60-sample pilot study of business simulation learners against which subsequent learners participating in online business simulation could be benchmarked. The results showed incremental improvement in the program due to changes made in assessment strategies, including the oral defense.
\end{abstract}


Keywords: Business Simulation, Benchmarking, Learning-by-doing, Factor Analysis, Path Analysis, Structural Equation Modeling (SEM)

\section{INTRODUCTION}

\section{Background of Research}

Building of dynamic capability, which is a strategic competency, is structurally modeled through a learning-by-doing approach via an online simulation game. A 60-participant pilot study explored perspectives of business simulation learners across three specializations, namely, Human Resources, General Business, and Accounting. The Cesim Global Strategy Simulation consisted of three practice turns and six to eight game turns, each equaling one year of real time. The scenario students played was based on a simulated Global handset market, managing, production, technological innovations, marketing, logistics, taxation, and finance of mobile handsets. The course team leader deployed Total Shareholder Return as the winning criterion, and this factor was based on an algorithm that accounted for net present value of dividends paid and the net increase in share valuation.

The analysis was based on the Dynamic Capabilities model proposed by Romme et al. (2010), and structural equation modeling (SEM) was used to fit and assess both 1) path analysis and 2) aggregated factor models that connected the students' experiential learning in a Cesim business simulation-based college course to the assessed components of the course. Path analysis implied causation, whereas the factor model was analyzed to determine the relationships among factors. Subsequent data, using the same Cesim simulation gaming platform, have enabled further validation of models proposed in this study and exploration of emergent properties, from incremental changes made to the assessed components of the course. Simulation gaming has the exciting potential to explore the ways in which educators in the digital age create a learning environment that attracts students' interest and engagement in learning strategic management (Miller, 2013).

\section{LITERATURE REVIEW}

The study of business simulation in the United Arab Emirates (UAE) has been limited. However, the Higher Colleges of Technology (UAE) has had recent deployment of another study about business strategy simulation. Using a qualitative phenomenological approach in a focus group setting, De Klerk (2015) reported that student perceptions of the use of a 
business simulation and students' lived experiences helped them understand and apply learning of business concepts. Kabeil (2009) developed a computer business simulation called the Strategic Management Game while teaching in the UAE at the University of Sharjah. The simulation had a logistics and manufacturing focus and comprised two products, tractors and trailers; over three available technology levels; and five teams called syndicates who played over five class meetings. Students participated in the simulation and filled human resources, production, inventory, marketing, and finance functions in the simulation. Kabeil (2009) measured the acquisition of business expertise in a 5-point Likert Scale to establish the learners' position on the Felder Index of Learning Style (ILS). Kabeil (2009) explored empowerment, dynamics, validity, intensity, holistic approach, and collective experience proposed in 2007 by Lainema and Lainema. Intensity and dynamics were the key factors developed using the business simulation.

Outside the UAE, Kiili (2007) also created a game and asserted that the three most important criteria in educational games were authenticity, collaboration, and learning by doing. Hoffman and Nadelson (2010) examined motivation to participate in simulation as rooted in gender, task orientation, time consumption, and socialization. On the other hand, Kilburn and Kilburn (2012) postulated that high levels of participation and interest, rather than teacher mentoring were more crucial for team success.

\section{THEORETICAL PERSPECTIVES}

Three theoretical perspectives were used in the analysis of benchmarking innovation education through business simulation. The linked feedback model by Romme et al. (2010) shown in Figure 1 guided the study while two models shown later in the results section of the study were synthesized as emergent properties of the pilot study. These were a recursive path analysis model that supported causation and a latent factor model that implied correlation through partial aggregation of factors.

\section{Dynamic Capabilities Model}

Constructs tested in two business simulation models were factors of students' experiential learning and assessment outcomes. Romme et al.'s (2010) theoretical perspective of the dynamic capability model guided the study using dynamic feedback loops of operating routines, knowledge articulation, and codification. 


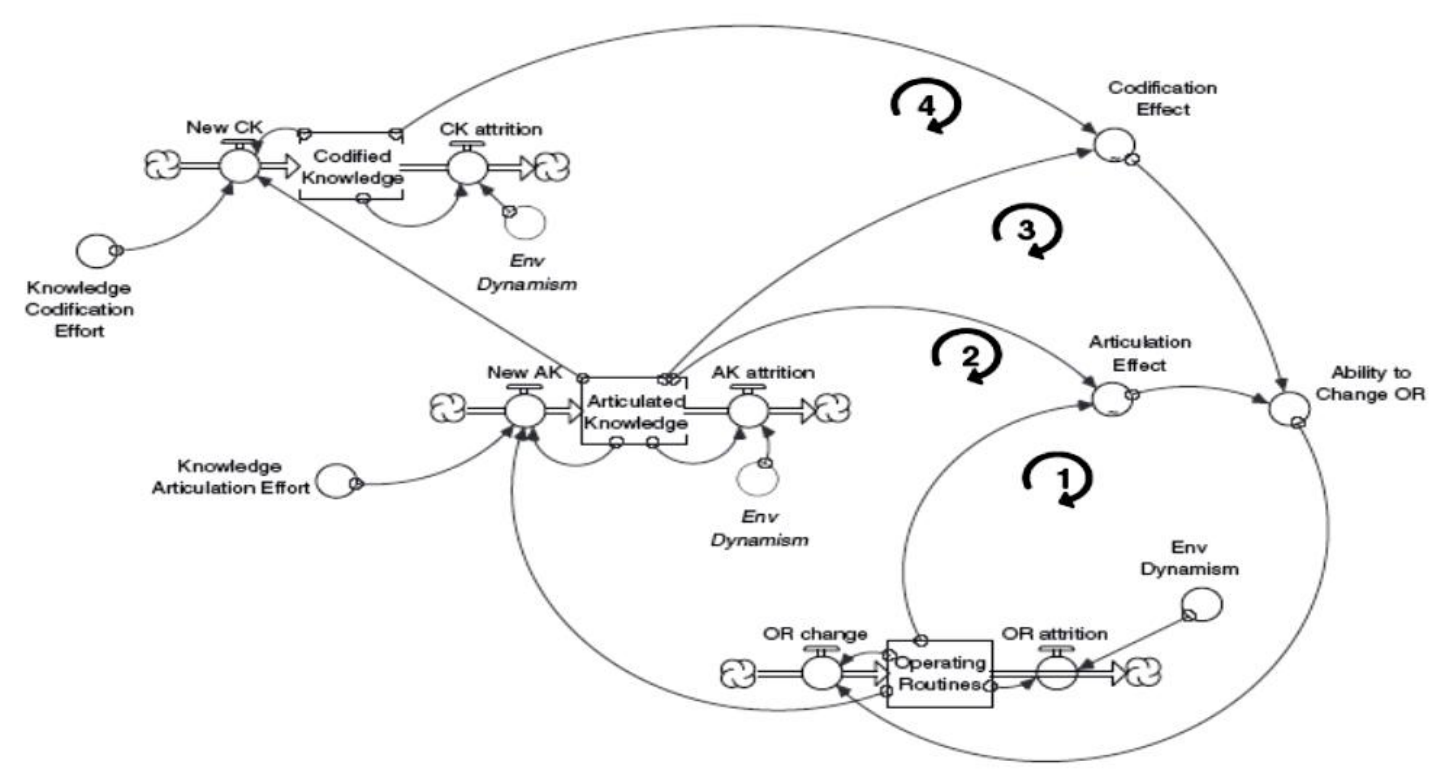

\section{Figure 1 Dynamic Capabilities Model of Deliberate Learning and Environmental Dynamism, Proposed by Romme et al. (2010)}

In the Cesim Simulation, operating routines were represented by procedural activities undertaken by teams to complete their game turn. Knowledge articulation described by Romme et al. (2010), was the manifestation of knowledge sharing that took place within the teams and amongst the competitors and included learners' individual expressions of learning via an assessed activity, the oral defense. Knowledge codification was the creation of written relics of the simulation, such as minutes of meetings, strategic planning documents, and written analyses of game situational outlooks and game turn outcomes. Operating routines were the learned procedures of students' experiential participation in the simulation.

\section{Field Testing}

The course leader and two instructors teaching the course at Higher Colleges of Technology in Fujairah met in the Spring of 2013 to discuss potential experiential factors present in the game and later, with students input, to identify factors that they perceived relevant to the game. The course leader utilized the findings from field-testing to develop a survey instrument that was reviewed by faculty teaching the course. The survey was 
comprised of eight items measured on a 5-point Likert scale and two categorical questions used to identify students by major, Accounting, Human Resources, or General Business, and by game Universe 1 or 2 . The objective was to determine whether the data could be used to support a theoretical model that demonstrated student experiential learning.

\section{Statement of Hypothesis}

Research questions and wording of each hypothesis centered on a measurement of the implied effects of variables for proposed inclusion in two theoretical models. Research Question 1 focused on the experiential factors used for path analysis and Research Questions 2 focused on the latent construct model. For path analysis, the research question and hypothesis were worded as follows:

Research Question Q1. To what extent, if any, does the proposed path analysis model imply causation related to student performance on assessments?

Hypothesis:

$\mathrm{H} 1^{0}$. The path analysis model is not related to assessment outcomes.

$\mathrm{H} 1^{\mathrm{a}}$. The path analysis model is related to assessment outcomes.

Research Question Q2. To what extent, if any, does the aggregated factor model connect the student's experiential learning in a business simulation-based college course to the assessed components of same the course?

Hypothesis:

$\mathrm{H} 2^{0}$. The aggregated factor model is not related to experiential learning. $\mathrm{H} 2^{\mathrm{a}}$. The aggregated factor model is related to experiential learning.

Experiential factors common to both path analysis and latent construct models were: Individual Role Effect on Company, Important Decision Making Areas, Preparation to Run Own Business, Team Placement, Perceived Realism, Functional Difficulty, and Functional Role. Factors that were structurally modeled in the latent construct model and part of the assessment outcomes in the path analysis were Coursework and Faculty-wide Assessment. The construct Final Grade was evaluated for fit in the latent construct model only.

\section{Recursive Path Diagram}

Path analysis yields a pattern that allows feedback and correlation; thus, it implies causation. Pilot results from the cause-effect path analysis model suggested that the simulation's experiential factors accounted for $19 \%$ of the variation in the coursework 
score and along with coursework, together these two factors accounted for $25 \%$ of the variation in Faculty-wide Assessment scores. The was inter-item correlations amongst the experiential factors indicated that four factors influenced Faculty-wide Assessment directly while five factors influenced Faculty-wide Assessment, through the coursework construct, as shown in Figure 2.

\section{Paradigm to Assess Learning in Business Simulation}

\section{A Recursive Path Diagram}

$$
\begin{aligned}
& \text { Chi-square }=3.308(14 \mathrm{df}) \\
& P=.998
\end{aligned}
$$

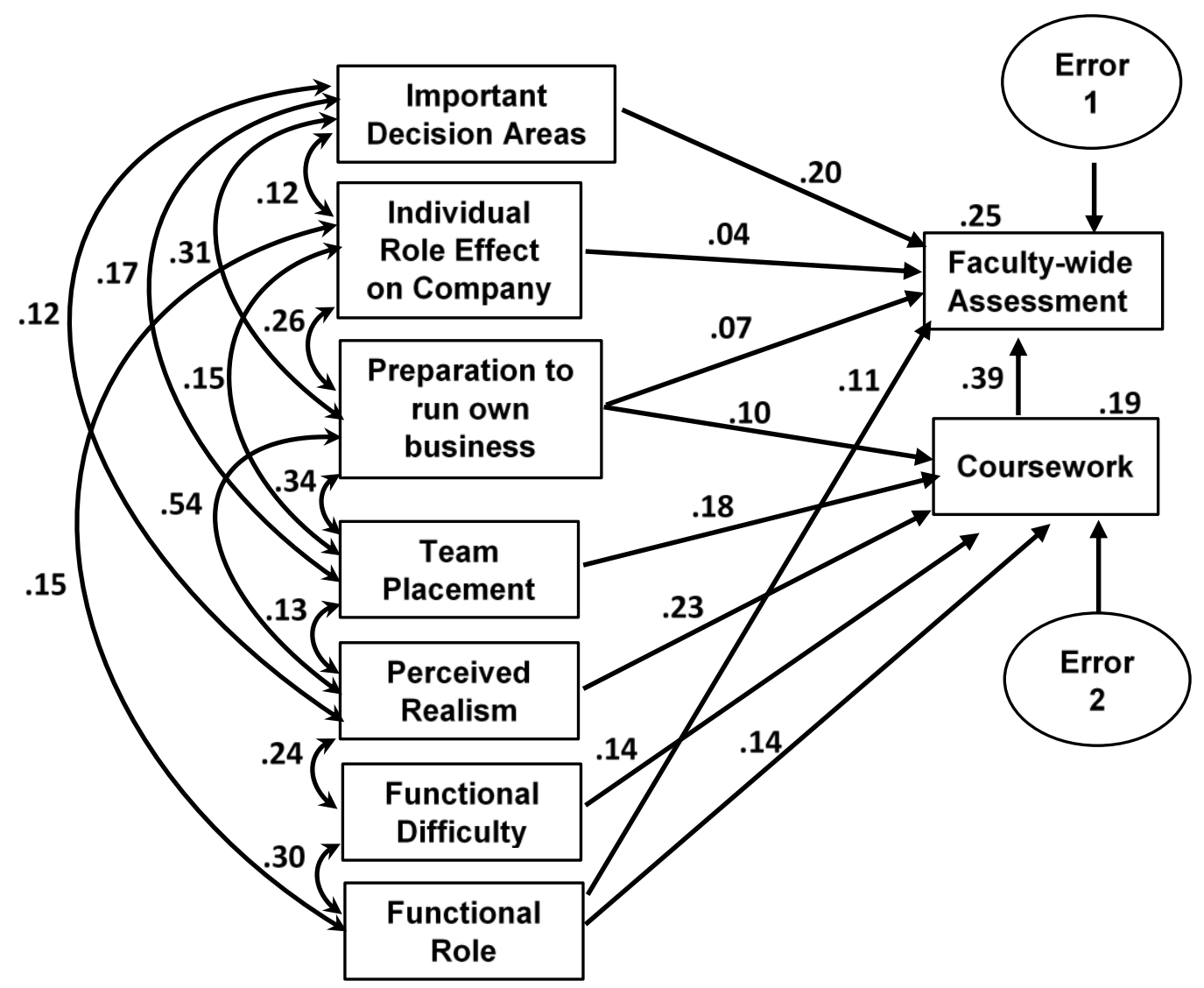

Figure 2 Business Simulation-Paradigm to Assess Learning (BS-PAL)

Path analysis goodness-of-fit was: $\chi^{2}=3.308 ; \chi^{2} / \mathrm{df}=.237$ where value $<5$ is acceptable but $<3$ is preferable; Goodness-of-Fit Index (GFI) $=.988$, where $>0.90$ is ideal; Adjusted Goodness-of-Fit Index $(\mathrm{AGFI})=.961$, where $>0.80$ is preferred; 
Comparative Fit Index $(\mathrm{CFI})=1.000$, where $>0.90$ is preferred; Root Mean Square Residuals $(\mathrm{RMSR})=.018$, where $<0.1$ and closer to 0 is preferred; Root Mean Square Error of Approximation (RMSEA) $=0.000$, where less than .080 is preferred; Normed Fit Index $(\mathrm{NFI})=.962$, where $>0.90$ is preferred; and Parsimony Normed Fit Index (PNFI) $=.374$, where $>0.60$ is preferred. The values were all in the acceptable range, as suggested by Garson (2008) and Kline (2012). Using the method of Garson (2008) to deconstruct the effects, the following direct effects were obtained: Important Decision Making Areas (ImpDA) > Faculty-wide Assessment $($ FWA $)=20 \%$, Individual Role Effect on Company (IREC) $>$ Faculty-wide Assessment $(F W A)=6 \%$, Probability to Run own Business $(\mathrm{PROB})>$ Faculty-wide Assessment $(\mathrm{FWA})=8 \%$, Coursework $(\mathrm{CW})>$ Faculty-wide Assessment $($ FWA $)=382$.

\section{Factor Analysis}

The ten construct model with three learning-based constructs and seven experientially based constructs initially produced a four factor rotated component matrix, as shown in Table 1 on the next page. Correlations among the seven experiential constructs indicated that original factors 2, 3, and 4 were collapsed into a single factor, as shown in Table 1 in the Two Factor Rotated Component Matrix. Coffman and McCallum (2005) advocated this approach to aggregate factors in structural equation modeling for models where empirical and/or qualitative evidence supports the validity of correlated factors and thus aggregation of two or more factors. In this case, the results from the recursive path model in Figure 2 supported empirically a partially aggregated latent variable model. Additionally, the qualitative factors of the Romme et al.'s (2010) model supported the face validity of the experiential factors. This yielded a partially aggregated latent variable model with adequate fit between the aggregated factors of student's simulation experience and learning assessment.

\section{Correlation Matrix and Latent Construct Model}

Table 2 below presents the correlation matrix showing the interrelationship between factors. Figure 3 on the following page displays the resulting latent factor model, which is based on the correlations in Table 2 and reflects inclusion of four factors aggregated into two factors. 
Table 1 Four Factor Rotation with Convergence after Six Rounds and the Aggregated Two-Factor Rotation Converging After Two Rounds

\begin{tabular}{lcccc}
\hline \multirow{2}{*}{ Four Factor Rotated Component Matrix } & \multicolumn{4}{c}{ Component } \\
\cline { 2 - 5 } & 1 & 2 & 3 & 4 \\
\hline Team Placement (TP) & & .327 & .476 & \\
Perceived Realism (PR) & .877 & & \\
Preparation To Run Own Business (PROB) & .651 & .562 & \\
Individual Role Effect On Company (IREC) & & .714 & \\
Importance Of Decision Area (ImpDA) & & & \\
Functional Role (FR) & & & .586 & .841 \\
Functional Difficulty (FD) & .382 & .586 & .709 \\
Coursework (CW) & .729 & .400 & & \\
Faculty-wide Assessment (FWA) & .901 & & & \\
Final & .980 & & & \\
\hline
\end{tabular}

Table 2 Correlation Matrix

\begin{tabular}{lcccccccccc}
\hline & TP & PR & PROB & IREC & ImpDA & CW & FR & FD & SWA & Final \\
\hline TP & 1.000 & .141 & $.338^{*}$ & .164 & .168 & $.245^{*}$ & .097 & .067 & .141 & $.208^{*}$ \\
PR & & 1.000 & $.543^{*}$ & -.035 & .096 & $.356^{*}$ & -.011 & $.272^{*}$ & .112 & $.232^{*}$ \\
PROB & & & 1.000 & $.261^{*}$ & $.308^{*}$ & $.286^{*}$ & .089 & .058 & $.260^{*}$ & $.314^{*}$ \\
IREC & & & & 1.000 & .133 & .024 & .162 & -.083 & .111 & .094 \\
ImpDA & & & & & 1.000 & .082 & .051 & -.061 & $.261^{*}$ & $.231^{*}$ \\
CW & & & & & & 1.000 & -.079 & .186 & $.422^{*}$ & $.731^{*}$ \\
FR & & & & & & & 1.000 & $.266^{*}$ & .099 & .041 \\
FD & & & & & & & & 1.000 & .057 & .121 \\
SWA & & & & & & & & & 1.000 & $.927^{*}$ \\
Final & & & & & & & & & & 1.000 \\
\hline
\end{tabular}

Note: $\left(^{*}\right) \mathrm{p}<0.05$ See Brown (2006). These are factor correlations in the latent model.

With the Partially Aggregated Parcel Latent Variable Model, goodness-of-fit (see Garson, 2008), $\chi^{2} / \mathrm{df}=.897$, where $<5$ is acceptable and $<3$ is preferred; Goodness-of-Fit Index $(\mathrm{GFI})=.907$, where $>0.90$ is preferred; Adjusted Goodness-of-Fit Index (AGFI) $=.850$, where $>0.80$ is preferred; Comparative Fit Index $(\mathrm{CFI})=1.000$, where $>0.90$ is preferred; Root Mean Square Residuals (RMSR) .094, where $<0.1$ and closer to 0 is preferred; Root Mean Square Error of Approximation (RMSEA) $=0.000$, where $<.080$ is preferred; Normed Fit Index $(\mathrm{NFI})=.962$ where $>0.90$ is preferred; and Parsimony Normed Fit Index $(\mathrm{PNFI})=.727$ where $>0.60$ is preferred See Garson (2008); Kenny (2012). 
Experiential Learning \& Assessment of Business Simulation A Latent Construct Model to Measure Building Dynamic Capability

Chi-square $=30.496(34 \mathrm{df})$ $\mathbf{P}=\mathbf{. 6 3 4}$

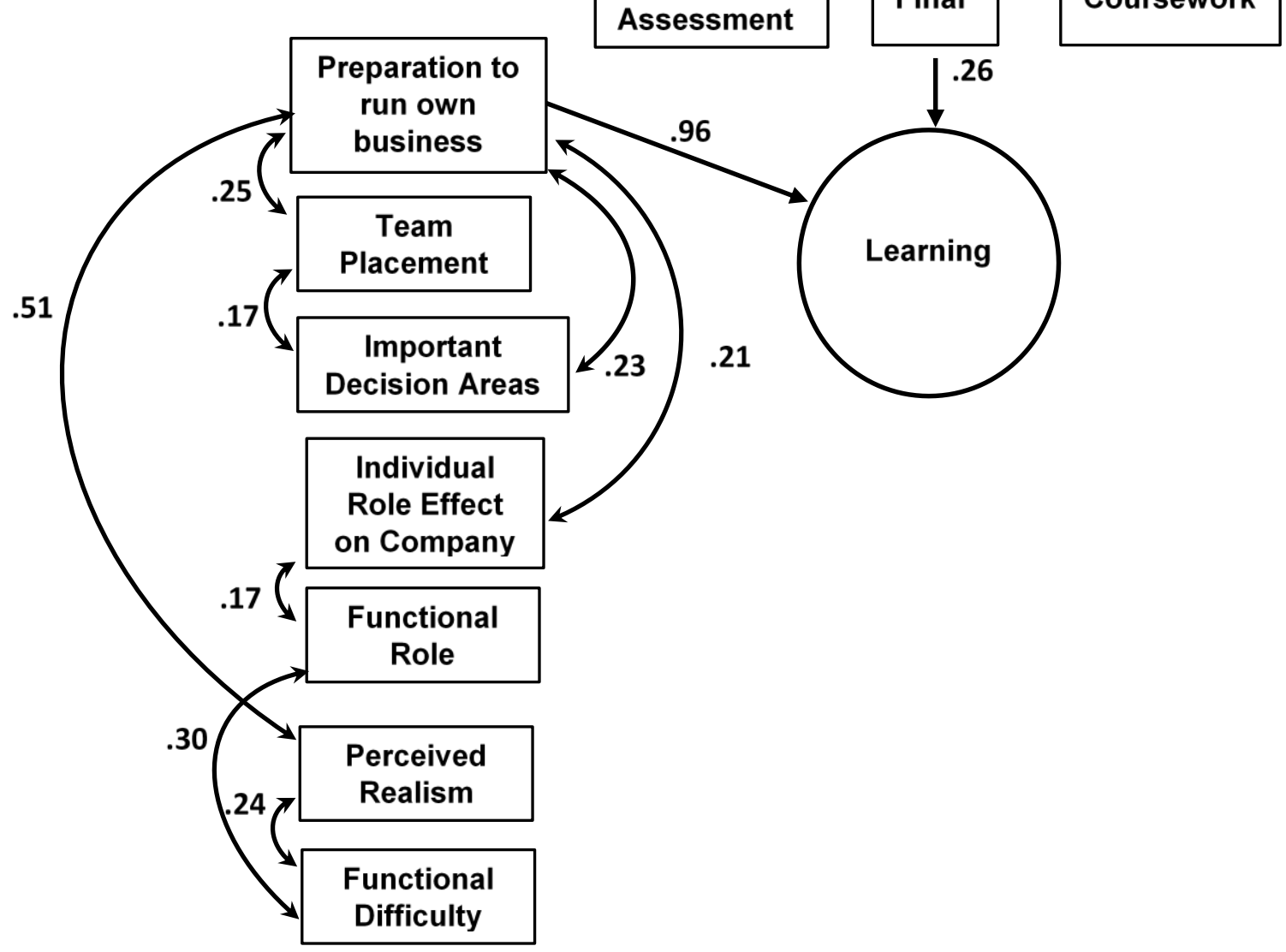

Figure 3 Dynamic Capabilities Experiential Learning and Assessment of Business Simulation (E-LABs) paradigm; proposed by Miller (2013) 


\section{NORMALITY, RELIABILITY AND STATISTICAL POWER}

Univariate and multivariate normality, reliability, statistical power, and correlation analyses were conducted examine the dataset. Statistical outcomes have greater credibility if these constraints are reported and are within parameters.

\section{Assessment of Normality}

With structural equation modeling, a robust research strategy includes an evaluation of normality. The Division of Statistics and Scientific Computation, College of Natural Sciences at the University of Texas in Austin (2013) provides recommendations to assess normality using IBM SPSS Amos 21. The data in the pilot study are distributed normally when multivariate kurtosis is negligible for most variables, as denoted by values less than 1.00; univariate skewness and kurtosis for variables cannot exceed +2.00 or drop below -2.00; otherwise, they should be investigated further for deviations from normality.

Table 3 Assessment of Normality (Path Analysis and Latent Construct Models)

\begin{tabular}{lrrrr}
\hline Variable & skew & c.r. & kurtosis & c.r. \\
\hline Functional Role & .490 & 1.549 & -.491 & -.776 \\
Functional Difficulty & -.589 & -1.862 & -.265 & -.420 \\
The Cesim Business Simulation is Realistic & -1.092 & $\mathbf{- \mathbf { 3 . 4 5 4 }}$ & .906 & 1.433 \\
Better Prepared To Run My Own Business & -1.144 & $\mathbf{- \mathbf { 3 . 6 1 6 }}$ & .738 & 1.167 \\
Understanding My Role Effect On Company & .295 & .933 & -1.429 & -2.260 \\
Team Placement Game Turn 8 & -.063 & -.199 & -1.196 & -1.891 \\
Important Decision Making Areas & .100 & .315 & -1.119 & -1.769 \\
Coursework & -.088 & -.278 & -.485 & -.766 \\
Faculty-wide Assessment & -.997 & $\underline{\mathbf{- 3 . 1 5 1}}$ & 1.541 & 2.436 \\
Final & -.828 & -2.617 & .840 & 1.329 \\
Multivariate Path Analysis & & & .670 & .185 \\
Multivariate Latent Construct & & & 33.088 & $\underline{\mathbf{8 . 2 7 2}}$ \\
\hline Not See Kly
\end{tabular}

Note: See Kline (2012).

To assess normality for SEM using the maximum likelihood estimator in factor models, a procedure that is robust to non-normality, Kline (2012) ignored the critical values generated by AMOS and referred to actual values of kurtosis, which must be $<8-10$, and skewness, which must be $<3$. Potential problems are underlined and in bold in Table 3 . The assessment of multivariate normality slightly exceeded the threshold of 8.0 , and 
Kolmogorov-Smirnov and Shapiro-Wilk results are shown in Table 4 where Functional Role showed the greatest deviation from normal.

\section{Table 4 Multivariate Normality Using Kolmogorov-Smirnov and Shapiro-Wilk Method}

\begin{tabular}{lcccccc}
\hline & \multicolumn{3}{c}{ Shapiro-Wilk } & \multicolumn{3}{c}{ Kolmogorov-Smirnov } \\
\cline { 2 - 6 } & statistic & df. & sig. & statistic & df. & sig. \\
\hline Functional Role (FR) & .099 & 60 & $\underline{. \mathbf{2 0 0} *}$ & .931 & 60 & .002 \\
Functional Difficulty (FD) & .105 & 60 & $\mathbf{. 0 9 7}$ & .951 & 60 & .018 \\
Final & .125 & 60 & .021 & .951 & 60 & .017 \\
Coursework (CW) & .157 & 60 & .001 & .962 & 60 & $\underline{\mathbf{. 0 5 8}}$ \\
Faculty-wide Assessment (FWA) & .105 & 60 & $\mathbf{. 0 9 6}$ & .941 & 60 & .006 \\
Importance Of Decision Area (ImpDA) & .160 & 60 & .001 & .962 & 60 & .001 \\
Team Placement (TP) & .118 & 60 & .038 & .919 & 60 & .001 \\
Perceived Realism (PR) & .287 & 60 & .000 & .808 & 60 & .000 \\
Preparation To Run Own Business (PROB) & .251 & 60 & .000 & .806 & 60 & .000 \\
Individual Role Effect On Company (IREC) & .282 & 60 & .000 & .826 & 60 & .000 \\
\hline Nonyyyyyy
\end{tabular}

Note: *. This is a lower bound of the true significance. Test of significance of non-normality, $p<.05$, a. Lilliefors significance correction.

\section{Reliability}

While validities of both path analysis and latent factor models were supported within the constraints identified, reliability was assessed at 6.99 for the relational factor model and 6.23 for the causative path analysis procedure using standardized Cronbach's $\alpha$, which indicates internal consistency of the instrument. Since items were standardized before being summed, it was appropriate to use the standardized measurement. Some researchers, such as Babbie (2010) proposed accepting lower Cronbach's $\alpha$ values, for inductive research in the social sciences when utilizing new instruments. However, these measurements do fall into the range of questionable 6.0-6.99, with greater than 7.0 being acceptable according to Kline (2012). Reliability may or may not improve with the additional 74 samples, which are already collected from learners for Spring 2015 semester, with additional sections being evaluated at the conclusion of the Fall 2015 and Spring 2016 semesters.

\section{Statistical Power}

The statistical power has been assessed a priori, suggesting that a sample size of 243 would be required to identify a medium effect with the path analysis model while $n=414$ would be required to have sufficient power with the latent construct model. In the present 
study, only a large effect could be identified with the path analysis model, with $\beta \geq .80$, and sample size is insufficient for the latent factor model. See Tables 5 and 6 for the protocols of power analysis (Faul et al., 2009). Replication of the study is required with either larger or multiple samples to demonstrate the stability of the results (Schreiber, et al., 2006).

Table 5 Protocol of Power Analysis: A Priori Medium Effect: Path Analysis Model

$\chi^{2}$ tests - Goodness-of-fit tests: Contingency tables

Analysis: A priori: Compute required sample size

Input: $\quad$ Effect size w (medium)

$=0.3$

$\alpha$ err prob

$=0.05$

Power (1 - $\beta$ err prob)

df

$=0.95$

Output: Noncentrality parameter $\lambda$

Critical $\chi^{2}$

$=21.8700000$

Total sample size

$=14.0671404$

Actual power

$=243$

$=0.9503250$

Table 6 Protocol of Power Analysis: A Priori Medium Effect: Latent Factor Model

\begin{tabular}{lll}
\hline$\chi^{2}$ tests - Goodness-of-fit tests: Contingency tables & \\
\hline Analysis: & A priori: Compute required sample size & \\
Input: & Effect size w (medium) & $=0.3$ \\
& $\alpha$ err prob & $=0.05$ \\
& Power $(1-\beta$ err prob) & $=34$ \\
& df & $=37.2600000$ \\
Output: & Noncentrality parameter $\lambda$ & $=48.6023674$ \\
& Critical $\chi^{2}$ & $=414$ \\
& Total sample size & $=0.9504153$ \\
& Actual power & \\
\hline
\end{tabular}

\section{APPLICATION OF THE BENCHMARKING MODEL}

The radar chart in Figure 4 provides an overview of the first semester and two successive semesters, and it is an example of one way to assess whether innovation education through business simulation has possibly occurred and whether improvements can be benchmarked. Total shareholder return (TSR) is reduced for the second two semesters, indicating more contested markets and providing less evidence of a team building a significant advantage. In the latter two learning cycles, students seemed somewhat better able to identify important decision making areas and their individual 
effects on the company, and they left the game with feelings of greater realism while feelings of the benefits of preparation to run their own business later increased. The only change other than different groups of students was a change in the Faculty-wide Assessment. The Faculty-wide Assessment has become an oral defense, instead of a written defense.

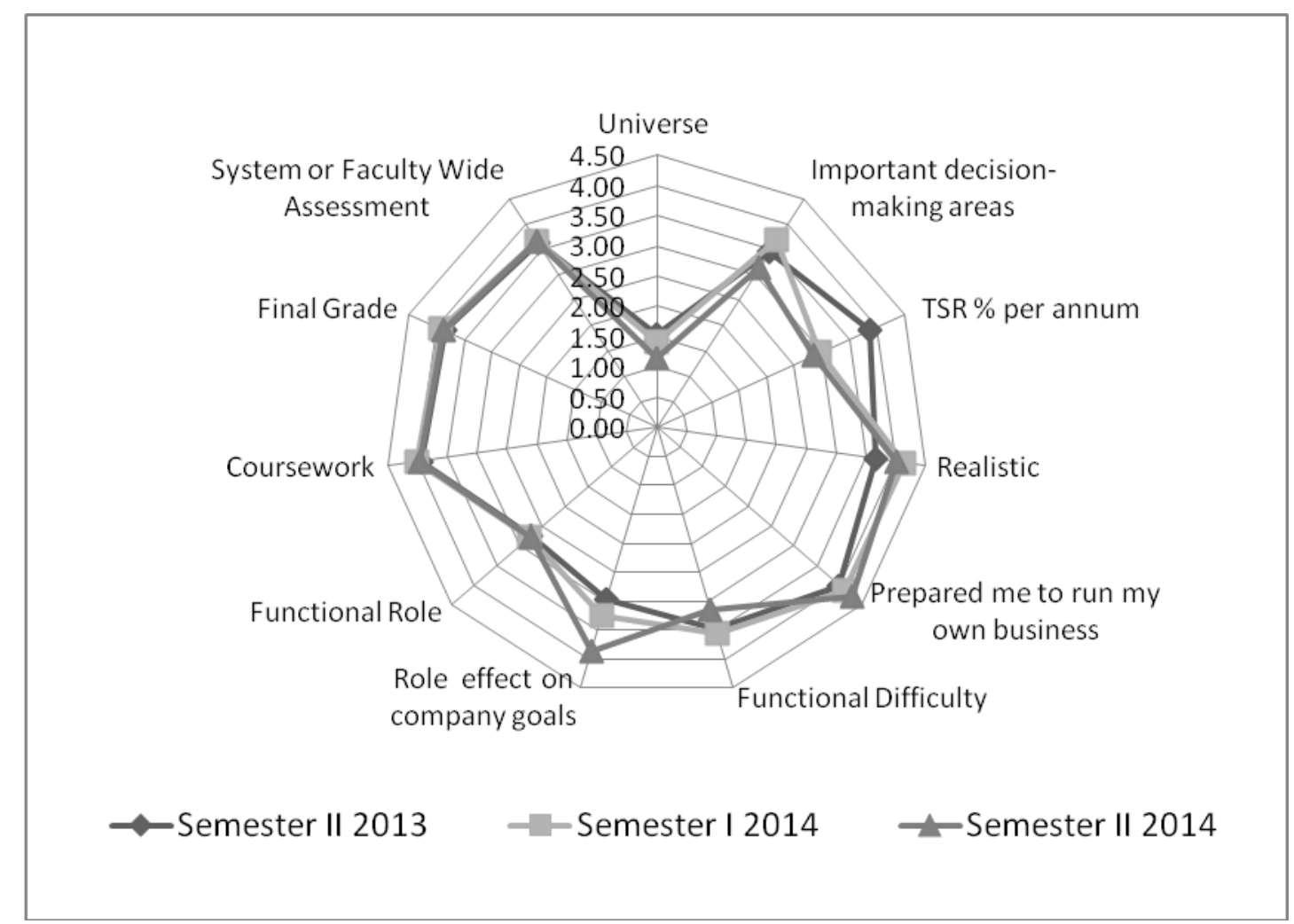

\section{Figure 4 Comparison of Business Simulation Student Outcomes across Three Semesters}

ACBSP's global business accreditation standards promote programs to demonstrate internal and external consistency, a particular challenge across larger multi-campus systems. The Higher Colleges of Technology, comprising 17 campuses and 240 business faculty, are thus motivated to form partnerships with other business programs to establish a collaboration program for external comparison of learning outcomes. International Council for Business Schools and Programs (ACBSP Region 8) where such partnerships are being explored includes GCC-based institutions from Kuwait, Qatar, Saudi Arabia and several from Europe and Ulaanbaatar, Mongolia. A partnership with Webster University of Athens 
in Greece was undertaken in April, 2015, with 26 students with chaperones completing an overseas study tour. However, this partnership was completed with Year 4 learners who had already completed the strategy simulation class. The context of the study was Integrative Industry Project, a capstone class. Collaboration between colleges has yet to be expanded to incorporate business strategy simulation.

\section{DISCUSSION AND CONCLUSION}

The constructs were validated in a pilot study comprised of 60 participants. It is proposed to augment this study with data from further business simulation students. The results demonstrated that Higher Colleges of Technology learners, displayed connections both among experiences and between factors. Their self-assessed learning-by-doing experiences being one aggregated factor, connected with the instructor assessed learning outcomes as being another.

Although the structural equation modeling procedures used were robust for non-normality, there were in both of the piloted models, instances of both univariate and multivariate non-normality. This demonstrated that normality was only partially satisfied, as about $10 \%$ of the tests statistics indicated that the data were not normally distributed. A power analysis was undertaken a priori that indicated the sample size was too small, however this was a pilot study. A larger study is proposed later with $n=450$ or greater number of participants collected during subsequent semesters to meet sample size criteria. Reliability of the instrument is in the questionable range, being $<0.7$ using a standardized Cronbach's alpha, with the latent factor model being just shy of acceptable at 0.699.

Additional data from the Spring 2015 Semester has been collected and data from Fall 2015 will be also included in subsequent reports presented at future conferences. A radar display of a subsequent data set from 2014 demonstrated a close overlay with some improvement over the results from the original piloting of the instrument. The results from the three assessed components were consistent across all three semesters, as was functional role, which indicated that no single student role in the game was overrepresented in the study. The only documented change was in the Faculty-wide Assessment instrument; being changed from a written defense to an oral defense. With this change, it was noted that self-reporting of experiential learning factors showed an incremental improvement. Did changes made to the Oral Defense increase student realization of greater experiential learning? 
Inferences about benchmarking innovation education could be made through business simulation by examining the Correlation Matrix in Table 7. The strongest factor correlations were between the Final Grade and Faculty or Faculty-wide Assessment at .927 and between Final Grade and Coursework at .731. Learner performance on the Oral Defense of the Faculty or Faculty-wide assessment was worth $30 \%$ of the Final Grade; thus, it had a greater influence compared to Coursework performance accounting for $70 \%$ of the Final Grade. Faculty-wide Assessment and Coursework were somewhat divergent in comparison to the other two assessment relationships, with a correlation of 422 .

Regarding the experiential factors, the correlations were .538 between Perceived Realism and Preparation to Run Own Business, .356 between Perceived Realism correlated and Coursework, .338 between Probability to Run Own Business and Team Placement correlated; .314 between Probability to Run Own Business and Final Grade, and .308 between Probability to Run Own Business and Important Decision Making Areas. Individual Role Effect on Company showed weak correlation with Functional Role at .162. Functional Role did not significantly correlate with any other factors, yet a better model fit was achieved when this factor was included in the model.

In conclusion, learners were given an opportunity to participate in a business simulation and learn innovation as part of the education process. The benchmarking process could be potentially expanded to other Higher Colleges of Technology campuses as well as other ACBSP Region 8 schools to provide a benchmarking tool usable for both internal and external benchmarking of the learning system that will motivate success in meeting Standard 4, i.e., Measurement and Analysis of Student Learning and Performance. The tool created based on this study enables an evaluation of innovation education through business strategy simulation to be benchmarked externally as well.

\section{ACKNOWLEDGMENT}

Thanks to Higher Colleges of Technology (HCT)-Fujairah Women's College, UAE; Cesim Oy, of Finland for support on the learning platform; the Accreditation Council of Business Schools and Programs (ACBSP), specifically Region 8, the International Council of Business Schools and Programs; the Gulf Comparative Education Society (GCES) and Rajamangala University of Technology, Krungthep; for supporting this research. 


\section{REFERENCES}

Babbie, E. (2010). The practice of social research. Belmont, California: Wadsworth Thomson Learning.

Brown, T.A. (2006). Confirmatory factor analysis for applied research. New York, NY: The Guilford Press.

Coffman, D.L., \& MacCallum, R.C. (2005). Using parcels to convert path analysis models into latent variable models. Multivariate Behavioral Research, 40(2), 235-259. http://dx.doi.org/10.1207/s15327906mbr4002_4.

De Klerk, J. (2015, April 6). Exploring the use of a business simulation to teach business concepts to Emirati student. Developments in Business Simulation and Experiential Learning, $42 . \quad$ Retrieved from https://journals.tdl.org/absel/index.php/absel/article/view/2909/2860.

Faul, F., Erdfelder, E., Buchner, A., \& Lang, A.-G. (2009). Statistical power analyses using G*Power 3.1: Tests for correlation and regression analyses. Behavior Research Methods, 41(4), 1149-1160. http://dx.doi.org/10.3758/BRM.41.4.1149.

Garson, D.G. (2013, November 24). Factor analysis: Statnotes. North Carolina State University Public Administration Program. Retrieved from http://faculty.chass.ncsu.edu/garson/PA765/index.htm.

Hoffman, B., \& Nadelson, L. (2010). Motivational engagement and video gaming: A mixed methods study. Educational Technology Research and Development, 58(3) 245-270.

Kabeil, M.M. (2009). The role of management games in mapping learning styles to elements of business know-how acquisition: A case study. Issues in Informing Science and Information Technology, 6, 531-543. Retrieved from http://www.iisit.org/Vol6/IISITv6p531-543Kabei1595.pdf.

Kiili, K. (2007). Foundation for problem-based gaming. British Journal of Educational Technology, 38(3), 394-404. http://dx.doi.org/10.1111/j.1467-8535.2007.00704.x.

Kline, R.B. (2012). Principles and practice of structural equation modeling (3rd ed.). New York, NY: Guilford.

Kilburn, B.R., \& Kilburn, A.J. (2012). The team vs. the individual: Login activity as a predictor of web-based simulation team success. Academy of Educational Leadership Journal, 16(3), 15-22.

Kenny, D. (2015, November 24). Measuring model fit. Retrieved from http://davidakenny.net/cm/fit.htm. 
Lainema, T., \& Lainema, K. (2007). Advancing acquisition of business know-how: Critical learning elements. Journal of Research on Technology in Education (JRTE), 40(2), 183-198. http://dx.doi.org/10.1080/15391523.2007.10782504.

Miller, A.H. (2013, November). Building dynamic capability: Modeling business simulation at a UAE Women's College. Paper presented at Engaged Learning in the Digital Age: International Council of Business Schools and Programs (ACBSP Region 8) and the 2013 ACBSP International Conference Brussels, Belgium. http://dx.doi.org/10.13140/RG.2.1.3951.4320.

Romme, A.G.L., Zollo, M., \& Berends, P. (2010). Dynamic capabilities, deliberate learning, and environmental dynamism: A simulation model. Industrial and Corporate Change, 19(4), 1-29. http://dx.doi.org/10.1093/icc/dtq031.

Schreiber, J.B. Stage, F.K., King, J., Nora A., \& Barlow, E.A. (2006). Reporting structural equation modeling and confirmatory factor analysis results: A review. The Journal of Educational Research, 323-338. http://dx.doi.org/10.3200/JOER.99.6.323-338.

The University of Texas at Austin (2016, April 6). To test for normality. Retrieved from http://ssc.utexas.edu/software/faqs/AMOS. 
